\title{
AN ALTERNATIVE METHOD FOR DETERMINING THE COEFFICIENT OF THERMAL EXPANSION OF COMPOSITE MATERIAL OF SPHERICAL PARTICLES
}

\author{
NGUYEN Dinh DuC \\ Vietnam National University, Hanoi \\ HoAng VAN TUNG \\ Hanoi Architectural University, Vietnam \\ Do ThanH Hang \\ National forecast center of hydrography, Vietnam.
}

\begin{abstract}
Composite material is widely used in modern structures. Many researchers have been involved in studying, developing and applying this kind of material. The composite material of spherical particles is a material composed of continuous matrix phase and spherical particles. In modern technique, it is very necessary to consider the influence of temperature on toughness and stability of structures. Therefore, determining the coefficient of thermal expansion of composite as a function of the coefficients and volume fractions of matrix and particle phases is a practical requirement. In this paper. we would like to introduce an alternative derivation method in order to obtain the thermal expansion coefficient of two-phase composite of spherical particles. Our results are the same as Vanin's and other authors'.
\end{abstract}

\section{INTRODUCTION}

Thermal expansion behaviour can be important when composite materials are used in conjunction with other materials and when it is necessary to match the thermal expansion coefficient of one structural component with another.

Among the kinds of composite material, particle-reinforced material is an important one. Many researchers have been involved in determining the effective thermal expansion coefficient of this kind of composite, especially composite of spherical inclusions [7].

Levin [8] showed that a simple relationship between the effective expansion coefficients and the effective elastic moduli could be derived for two-phase materials using the thermoelastic stress-strain relations. For an isotropic composite with two isotropic phases the basic relationship can be written in form

$$
\left.\alpha^{*}=\bar{\alpha}+\frac{\alpha_{1}-\alpha_{2}}{\left[\left(1 / K_{1}\right)-\left(1 / K_{2}\right)\right.}\right]\left[\frac{1}{K^{*}}-\overline{\left(\frac{1}{K}\right)}\right],
$$

where $\alpha_{1}, \alpha_{2}$ are the linear thermal expansion coefficients of the two phases, $K_{1}, K_{2}$ are their bulk moduli, $K^{*}$ and $\alpha^{*}$ the effective bulk modulus and effective thermal expansion coefficient of composite, and the bars over the symbols indicate volume averages.

If one of the phases is a fluid (or a low shear modulus matrix) and the other consists of rigid particles, mechanical interactions represented by the final term of (1.1) can be 
neglected

$$
\frac{1}{K *} \simeq \overline{\left(\frac{1}{K}\right)} \equiv \frac{1}{K_{1}} V_{1}+\frac{1}{K_{2}} V_{2}
$$

where $V_{1}, V_{2}$ are the volume fractions of phases and it follows from equation (1.1) the simple rule of mixture

$$
\alpha^{*} \simeq \bar{\alpha} \equiv \alpha_{1} V_{1}+\alpha_{2} V_{2} .
$$

For a dilute suspension in which spherical particles with bulk modulus $K_{1}$ are dispersed in a matrix with bulk modulus $K_{2}$ and shear modulus $\mu_{2}$, we can use Hashin's relation for effective bulk modulus as follows [1].

$$
K^{*}=K_{2}+\left(K_{1}-K_{2}\right) \frac{3 K_{2}+4 \mu_{2}}{3 K_{1}+4 \mu_{2}} V_{1} .
$$

This relation may be expected to apply when the volume fraction of particles $V_{1}$ does not exceed a few percent.

From equations (1.1) and (1.4) we obtain

$$
\alpha^{*}=\alpha_{2}+\frac{\left(\alpha_{1}-\alpha_{2}\right) K_{1}\left(3 K_{2}+4 \mu_{2}\right) V_{1}}{K_{2}\left(3 K_{1}+4 \mu_{2}\right)+\left(K_{1}-K_{2}\right)\left(3 K_{2}+4 \mu_{2}\right) V_{1}} .
$$

For higher particle concentrations, Hashin obtained the exact relation for the effective bulk modulus of this system [1]

$$
K^{*}=K_{2}+\left(K_{1}-K_{2}\right) \frac{\left(3 K_{2}+4 \mu_{2}\right)}{3 K_{1}+4 \mu_{2}+3\left(K_{2}-K_{1}\right) V_{1}} .
$$

Subsituting (1.6) in Levin's equation (1.1), we obtain the relation

$$
\alpha^{*}=\alpha_{2}-\frac{\left(\alpha_{2}-\alpha_{1}\right) K_{1}\left(3 K_{2}+4 \mu_{2}\right) V_{1}}{K_{2}\left(3 K_{1}+4 \mu_{2}\right)+4\left(K_{1}-K_{2}\right) \mu_{2} V_{1}} .
$$

This relation can also be obtained from the expressions given by Kerner [7].

Using the relationships

$$
K=\frac{E}{3(1-2 \nu)}, \quad \mu=\frac{E}{2(1+\nu)}
$$

equation (1.7) can be written in the form

$$
\alpha^{*}=\alpha_{2}-\left(\alpha_{2}-\alpha_{1}\right) \frac{3\left(E_{1} / E_{2}\right)\left(1-\nu_{2}\right) V_{1}}{\left(E_{1} / E_{2}\right)\left[2 V_{1}\left(1-2 \nu_{2}\right)+\left(1+\nu_{2}\right)\right]+2\left(1-2 \nu_{1}\right) V_{2}}
$$

which corresponds with the relations obtained by Fahmy and Ragai [6]. These two authors derived equation (1.8) from a consideration the difference between linear strains at interfaces in a composite sphere.

Assuming homogeneous strain throughout the composite and using a balance of internal average stresses, Turner suggested thermal expansion coefficient of two-phase composite taking into account the stress interaction between phases as follows.

$$
\alpha^{*}=\frac{K_{1} \alpha_{1} V_{1}+K_{2} \alpha_{2} V_{2}}{K_{1} V_{1}+K_{2} V_{2}}=\frac{\overline{K \alpha}}{\bar{K}} .
$$


Employing extremum principles of thermoelasticity and taking into account the stress interaction between components, Schapery has derived the following relation for the thermal expansion coefficient of composite material

$$
\alpha^{*}=\alpha_{1}+\left(\alpha_{2}-\alpha_{1}\right) \frac{\left(1 / K^{*}\right)-\left(1 / K_{1}\right)}{\left(1 / K_{2}\right)-\left(1 / K_{1}\right)}
$$

Finally, we discuss about Vanin's derivation [2]. According to Eshelby's formula [1], the energy of composite material of spherical particles is given as follows

$$
U=U_{0}+\frac{1}{2} \iint_{S_{i}}\left(\sigma_{i} u_{i}^{0}-\sigma_{i}^{0} u_{i}\right) d S,
$$

where $S_{i}$ is the surface of spherical particles, $\left(\sigma_{i}, u_{i}\right)$ - the stresses and displacements of composite and $\left(\sigma_{i}^{0}, u_{i}^{0}\right)$ the stresses and displacements of homogeneous elastic material. Fundamentally, using this energy relation Vanin obtained the thermal expansion coefficient of two-phase composite material as follows

$$
\beta^{*}=\beta_{2}-\left(\beta_{2}-\beta_{1}\right) \frac{\xi K_{1}\left(3 K_{2}+4 G_{2}\right)}{3 K_{1} K_{2}+4 G_{2}\left[\xi K_{1}+(1-\xi) K_{2}\right]} .
$$

Nevertheless, the displacement field selected by Vanin is relatively complex.

At present work, we would like to introduce an alternative method of derivation in order to give the effective thermal expansion coefficient of two-phase composite of spherical particles. Our derivation is based on the theory of thermo-elasticity and broadenning results of Lame problem taking into account the influence of temperature [3]. Our results coincide with relations obtained by Vanin, Hashin, Kerner, Fahmy and Ragai.

\section{THE PROBLEM}

Investigating composite material of spherical particles is derived by considering a representative volume element having form of a sphere contained in a cube. According to composite spheres model suggested by Hashin [1], the outer cube is estimated by a sphere having the same volume. This model give us a solid sphere covered by a spherical shell. Inner solid sphere represents particle phase, whereas outer spherical shell- matrix phase. We assume that particle and matrix phases are made of isotropic homogeneous elastic materials, moreover, the radii of particles are the same. Consequently, present problem can be posed as follows.

Let us consider a heterogeneous sphere that core sphere $(0 \preceq r \preceq a)$ and spherical shell $(a \preceq r \preceq b)$ are homogeneous materials of properties $\left(\lambda_{1}, \mu_{1}\right),\left(\lambda_{2}, \mu_{2}\right)$ and thermal expansion coefficients $\left(\alpha_{1}, \alpha_{2}\right)$, respectively. It is supposed that the heterogeneous sphere is subjected to a hydrostatic stress $p_{2}$ on the boundary $r=b$, at the same time, is warmed up by temperature $T$ (in fact, temperature interval $\Delta T=T-T_{0}$ represents the difference of temperatures and $T_{0}$ is initial temperature). There exists a problem to think of proposed inhomogeneous sphere as a composite sphere and determine the effective thermal expansion coefficient of composite as a function of coefficients and elastic moduli of constituent phases. 


\section{GOVERNING RELATIONS}

Some governing relations of proposed problem are given in the spherical coordinate system as follows [3].

Because of symmetry, it is clear that the only non-zero displacement component is radius displacement $u_{r}$. Moreover, it is a function of radius $r$.

$$
u_{r}=u_{r}(r), u_{\theta}=u_{\varphi}=0 .
$$

By theory of thermoelasticity [3], we express the components of stress tensor in terms of displacements.

$$
\begin{gathered}
\sigma_{r r}=\lambda \theta+2 \mu \varepsilon_{r r}-(3 \lambda+2 \mu) \alpha \Delta T, \\
\sigma_{\theta \theta}=\sigma_{\varphi \varphi}=\lambda \theta+2 \mu \varepsilon_{\varphi \varphi}-(3 \lambda+2 \mu) \alpha \Delta T .
\end{gathered}
$$

Here $\theta=d u_{r} / d r+2 u_{r} / r$ and $\Delta T=T-T_{0}$ considered as a constant in present work.

Putting (3.2) into equilibrium equation

$$
\frac{d \sigma_{r r}}{d r}+\frac{2}{r}\left(\sigma_{r r}-\sigma_{\varphi \varphi}\right)=0
$$

we obtain the following differential equation for $u_{r}$

$$
\frac{d^{2} u_{r}}{d r^{2}}+\frac{2}{r} \frac{d u_{r}}{d r}-2 \frac{u_{r}}{r^{2}}=0 .
$$

\section{SOLUTION METHOD}

As was mentioned above, the governing idea for solving recommended problem is that widenning results of Lame problem when consider the influence of temperature. Firstly, we separately define the state of displacement and stress of particle and matrix phases of heterogeneous sphere. Then we define displacement and stress fields of composite sphere. By a fact that displacement on boundary of composite sphere is the same as that on boundary of matrix spherical shell, we obtain the objective of problem.

\subsection{Part of matrix phase}

In part of matrix phase ( $a \preceq r \preceq b$ ) the displacement and stress are in forms

$$
\begin{gathered}
u_{r}^{(2)}=A_{2} r+\frac{B_{2}}{r^{2}}, \\
\sigma_{r r}^{(2)}=\left(3 \lambda_{2}+2 \mu_{2}\right) A_{2}-\frac{4 B_{2} \mu_{2}}{r^{3}}-\left(3 \lambda_{2}+2 \mu_{2}\right) \alpha_{2} \Delta T .
\end{gathered}
$$

Introduction of $\sigma_{r r}^{(2)}$ into boundary and surface conditions

$$
\left.\sigma_{r r}^{(2)}\right|_{r=b}=-p_{2},\left.\quad \sigma_{r r}^{(2)}\right|_{r=a}=-p,
$$

(in fact, $p$ is interaction stress between two phases), we define integration constants as well as displacement and stress in the matrix phase

$$
\begin{aligned}
& u_{r}^{(2)}=\left[\frac{p a^{3}-p_{2} b^{3}}{b^{3}-a^{3}} \frac{1}{3 \lambda_{2}+2 \mu_{2}}+\alpha_{2} \Delta T\right] r+\frac{\left(p-p_{2}\right) a^{3} b^{3}}{\left(b^{3}-a^{3}\right) 4 \mu_{2}} \frac{1}{r^{2}}, \\
& \sigma_{r r}^{(2)}=\frac{p a^{3}-p_{2} b^{3}}{b^{3}-a^{3}}+\frac{\left(p_{2}-p\right) a^{3} b^{3}}{b^{3}-a^{3}} \frac{1}{r^{3}}+\left(3 \lambda_{2}+2 \mu_{2}\right) \alpha_{2} \Delta T .
\end{aligned}
$$




\subsection{Part of particle phase}

In this part $(0 \preceq r \preceq a)$ displacement and stress fields have form

$$
\begin{gathered}
u_{r}^{(1)}=A_{1} r, \\
\sigma_{r r}^{(1)}=\left(3 \lambda_{1}+2 \mu_{1}\right) A_{1}-\left(3 \lambda_{1}+2 \mu_{1}\right) \alpha_{1} \Delta T .
\end{gathered}
$$

By the continuity conditions of displacement and stress at interface $r=a$

$$
u_{r}^{(+)}=u_{r}^{(-)}, \quad \sigma_{r r}^{(+)}=\sigma_{r r}^{(-)},
$$

we obtain the following relations of $A_{1}$ and interaction stress $p$.

$$
\begin{gathered}
A_{1}=-\frac{\left(K_{2}+4 \mu_{2} / 3\right) p_{2} b^{3}-\left[\left(3 K_{2} b^{3}+4 \mu_{2} a^{3}\right) K_{1} \alpha_{1}+4 \mu_{2}\left(b^{3}-a^{3}\right) K_{2} \alpha_{2}\right] \Delta T}{\left(3 K_{2} b^{3}+4 \mu_{2} a^{3}\right) K_{1}+4 \mu_{2}\left(b^{3}-a^{3}\right) K_{2}}, \\
p=-K_{1} \frac{\left(3 K_{2}+4 \mu_{2}\right) p_{2} b^{3}-12 \mu_{2} K_{2}\left(b^{3}-a^{3}\right)\left(\alpha_{2}-\alpha_{1}\right) \Delta T}{\left(3 K_{2} b^{3}+4 \mu_{2} a^{3}\right) K_{1}+4 \mu_{2}\left(b^{3}-a^{3}\right) K_{2}}
\end{gathered}
$$

where $K_{i}=\lambda_{i}+2 \mu_{i} / 3 \quad(i=1,2)$.

\subsection{Equivalent homogeneous sphere}

Now we consider equivalent homogeneous sphere having radius $r=b$, properties $(\lambda, \mu)$ and thermal expansion coefficient $\alpha$, such a sphere is referred to as a composite sphere. Composite sphere is subjected to a hydrostatic stress $p_{2}$ on boundary $r=b$ and is warmed up by temperature $T$.

Because the composite sphere is solid, solution form is similar to that of particle phase.

$$
\begin{gathered}
u_{r}^{*}=A^{*} r, \\
\sigma_{r r}^{*}=(3 \lambda+2 \mu) A^{*}-(3 \lambda+2 \mu) \alpha \Delta T .
\end{gathered}
$$

The displacement field is defined from boundary condition

$$
\left.\sigma_{r r}^{*}\right|_{r=b}=-p_{2},
$$

as follows

$$
u_{r}^{*}=\left[\frac{-p_{2}}{3 \lambda+2 \mu}+\alpha \Delta T\right] r
$$

In fact, the displacements of composite sphere and spherical matrix shell are the same, specifically

$$
\left.u_{r}^{(2)}\right|_{r=b}=\left.u_{r}^{*}\right|_{r=b} .
$$

Putting equations (4.4) and (4.12) into equation (4.13) taking into account (4.9), we obtain the following equation

$$
\begin{aligned}
& p_{2}\left[\left(K+4 \mu_{2} / 3\right)\left(K_{1}-K_{2}\right) a^{3}-\left(K-K_{2}\right)\left(K_{1}+4 \mu_{2} / 3\right) b^{3}\right]+ \\
& +\left[K K_{2}\left(3 K_{1}+4 \mu_{2}\right)\left(\alpha-\alpha_{2}\right)\left(a^{3}-b^{3}\right)-K K_{1}\left(3 K_{2}+4 \mu_{2}\right)\left(\alpha-\alpha_{1}\right) a^{3}\right] \Delta T=0 .
\end{aligned}
$$

Equation (4.14) is satisfied for arbitrary $p_{2}$, therefore, its coefficients must be zero. Equating to zero the coefficient of $p_{2}$ gives us relation

$$
K=K_{2}+\frac{\left(K_{1}-K_{2}\right) \xi}{1+\left(K_{1}-K_{2}\right)\left(K_{2}+4 G_{2} / 3\right)^{-1}(1-\xi)},
$$


which is the same as Hashin's formula introduced in Christensen's work [1] for determining the effective bulk modulus of composite of spherical particles.

Then making the term independent of $p_{2}$ to be zero, we obtain relation

$$
\alpha=\alpha_{2}+\left(\alpha_{1}-\alpha_{2}\right) \frac{K_{1}\left(3 K_{2}+4 G_{2}\right) \xi}{K_{2}\left(3 K_{1}+4 G_{2}\right)+4\left(K_{1}-K_{2}\right) G_{2} \xi}
$$

where $\xi=\frac{a^{3}}{b^{3}}$ is the volume fraction of particle phase.

We readily recognize that relation (4.16) is the same as Vanin's formula (1.12) for calculating the effective thermal expansion coefficient of composite material of sphericai particles. It is a function of the coefficients, elastic moduli and volume fractions of two phases.

\section{CONCLUSIONS}

By composite spheres model and using the theory of thermoelasticity, we have determined the effective thermal expansion coefficient of two-phase composite material of sphercial reinforced particles. Our formula coincide with result obtained by Vanin and several other authors such as Hashin, Kerner, Fahmy and Ragai. Mathematically, we think that our derivation method is more simple than Vanin's and other authors'. Moreover, our results exactly represent the nature of heat transfer, specifically, the effective bulk modulus of composite is obtained as a corollary. Finnally, this approach can be broadenned to composite cylinders model in order to reach the thermal expansion coefficients of fibre-reinforced composite material.

Acknowledgment. This publication is completed with financial support of National Council for Natural Sciences.

\section{REFERENCES}

1. R. M. Christensen, Mechanics of Composite Materials, John Wiley and Sons Inc, New York, 1979.

2. G. A. Vanin, Micro - Mechanics of Composite Materials, Naukova dumka, Kiev, 1985.

3. Dao Huy Bich, The Theory of Elasticity, The Publishing House of Vietnam National University, Hanoi, 2001.

4. Nguyen Hoa Thinh, Nguyen Dinh Duc, The Composite Material-Mechanics and Technology, The Publishing House of Science and Technique, Hanoi, 2002.

5. S. Lemieux, S. Elomari, J. A. Nemes, M. D. Skibo, Thermal expansion of isotropic Duralcan metal-matrix composites, Journal of Materials Science 33 (1998) 4381-4387.

6. A. A. Fahmy, A. N. Ragai, Thermal expansion behaviour of two-phase solids, Journal of Applied Physics 41 (13) (1970) 51085111.

7. D. K. Hale, Review the physical properties of composite materials, Journal of Materials Science 11 (1976) 2105-2141.

8. V. M. Levin, On the coefficients of thermal expansion of heterogeneous materials, Mekh. Tverd Tela (in Russian) $\mathbf{8 8}$ (1968).

9. B. W. Rosen and Z. Hashin, Effective thermal expansion coefficients and specific heats of composite materials, Int. J. Eng. Sci. 8 (157) (1970) 157 - 173. 


\section{MỘT PHƯƠNG PHÁP KHÁC DÊ XÁC ĐỊNH HỆ SỐ GIÃN NỞ NHIỆT CƯA VẬT LIỆU COMPOSITE VỚI CÁC HẠT ĐƠN CẦU}

Vật liệu composite được sử dụng rộng rãi trong nhiều kết cấu hiện đại nhờ tính ưu việt của nó. Việc nghiên cứu, phát triển và ứng dụng loại vật liệu này nhận được sự quan tâm của nhiều nhà nghiên cứu. Vật liệu composite với các hạt độn cầu là một loại vật liệu đằng hướng được cấu thành từ pha nền liên tục và các hạt độn cầu. Trong kỹ thuật hiện đại, việc xem xét ành hường của nhiệt độ đến độ bền và độ ởn định của các kết cấu là rất cần thiết. Do đó việc xác định hệ số dãn nớ nhiệt của composite như một hàm của các hệ số dãn nở nhiệt và tỷ lệ thể tích của các vật liệu thành phần là một yêu cầu rất thực tế. Trong bài báo này chúng tôi giới thiệu một phương pháp khác nhằm thu được hệ số dãn nở nhiệt cúa composite hai pha độn các hạt cầu. Kết quá. của chúng tôi trùng hoàn toàn với kết quả của Vanin và một số tác giả khác. 